\title{
Counting rational points on threefolds
}

\author{
Niklas Broberg* Per Salberger
}

July 24, 2003

\begin{abstract}
Let $X \subset \mathbb{P}^{4}$ be an irreducible hypersurface and $\varepsilon>0$ be given. We show that there are $O\left(B^{3+\varepsilon}\right)$ resp. $O\left(B^{55 / 18+\varepsilon}\right)$ rational points on $\mathbb{P}^{4}$ lying on $X$ when $X$ is of degree $d \geq 4$ resp. $d=3$. The implied constants depend only on $d$ and $\varepsilon$.
\end{abstract}

\section{Introduction}

Let $\overline{\mathbb{Q}}$ be an algebraic closure of $\mathbb{Q}$ and $\mathbb{P}^{n}$ be projective $n$-space over $\overline{\mathbb{Q}}$. That is, $\mathbb{P}^{n}$ is the set of one-dimensional linear subspaces of $\overline{\mathbb{Q}}^{n+1}$. A point on $\mathbb{P}^{n}$ is said to be rational if it represents a subspace of $\overline{\mathbb{Q}}^{n+1}$ generated by an element in $\mathbb{Q}^{n+1}$. The height of a rational point $p \in \mathbb{P}^{n}(\mathbb{Q})$ is given by

$$
H(p)=\max \left(\left|Z_{0}\right|, \ldots,\left|Z_{n}\right|\right),
$$

where $Z_{0}, \ldots, Z_{n}$ are relatively prime integers such that $p=\left[Z_{0}, \ldots, Z_{n}\right]$.

For a Zariski closed subset $X$ of $\mathbb{P}^{n}$, let $N(X, B)$ be the counting function

$$
N(X, B)=\#\left\{p \in \mathbb{P}^{n}(\mathbb{Q}) \cap X: H(p) \leq B\right\},
$$

where $\mathbb{P}^{n}(\mathbb{Q})$ is the set of rational points on $\mathbb{P}^{n}$. In the case where $X$ is defined by an irreducible form $F\left(Z_{0}, \ldots, Z_{n}\right)$ of degree $d \geq 2$, Heath-Brown [4] conjectured that

$$
N(X, B)=O_{n, d, \varepsilon}\left(B^{n-1+\varepsilon}\right)
$$

for any $\varepsilon>0$. The implied constant should thus not depend on $F$, only on $n, d$, and $\varepsilon$. In the same paper he verified the conjecture for curves, surfaces, and for quadrics of any dimension. In [1], Browning proved the conjecture for non-singular hypersurfaces in $\mathbb{P}^{4}$ of degree at least four. In this paper we shall prove the following result.

Theorem 1. Let $X \subset \mathbb{P}^{4}$ be a hypersurface defined by an irreducible form $F\left(Z_{0}, \ldots, Z_{n}\right)$ with coefficients in $\overline{\mathbb{Q}}$. Then the following holds for any $\varepsilon>0$ :

$$
N(X, B)= \begin{cases}O_{d, \varepsilon}\left(B^{3+\varepsilon}\right) & \text { if } d \geq 4 \\ O_{\varepsilon}\left(B^{55 / 18+\varepsilon}\right) & \text { if } d=3 .\end{cases}
$$

${ }^{*}$ While working on this paper, the author was supported by the EC network Arithmetic Algebraic Geometry. 


\section{Preliminaries}

In this section we recollect some known estimates of counting functions. We also state and prove some results that we use in the proof of Theorem 1 . First note that:

- We follow the convention that a subvariety $X$ of $\mathbb{P}^{n}$ is a closed subset that is not necessarily irreducible. A hypersurface of $\mathbb{P}^{n}$ is a subvariety of codimension one. All varieties are defined over $\overline{\mathbb{Q}}$.

- We shall often count rational points of bounded height on $\mathbb{P}^{n}$ which lie on $X$ even if $X$ is not defined over $\mathbb{Q}$. The same situation occur for subvarieties of the dual space $\mathbb{P}^{n *}$ of $\mathbb{P}^{n}$ when we count hyperplanes $\Gamma$ of $\mathbb{P}^{n}$ defined over $\mathbb{Q}$ for which $\Gamma \cap X$ is reducible. We shall also use coordinates over $\mathbb{Q}$ for Grassmannians.

- If $X \subset \mathbb{P}^{n}$ is a hypersurface, then we define the degree of $X$ to be the degree of the corresponding reduced scheme. That is, we let $d=\operatorname{deg}(X)$ be the minimal degree among all forms defining $X$. This implies that an intersection $\Lambda \cap X$ with a linear subspace $\Lambda \subset \mathbb{P}^{n}$ not contained in $X$ may have lower degree than $X$.

- Our calculations involve numerous constants. To avoid introducing the constants explicitly, we use the following notation.

Suppose that $f_{1}$ and $f_{2}$ are functions such that $f_{i}(B) \geq 0$ for all $B \geq 1$. We write

$$
f_{1}(B) \ll_{p_{1}, \ldots, p_{k}} f_{2}(B),
$$

if there exists a positive constant $C$, depending only on the parameters $p_{1}, \ldots, p_{k}$, such that $f_{1}(B) \leq C f_{2}(B)$ for all $B \geq 1$. We write

$$
f_{1}(B) \asymp_{p_{1}, \ldots, p_{k}} f_{2}(B),
$$

if $f_{1}(B) \ll_{p_{1}, \ldots, p_{k}} f_{2}(B)$ and $f_{2}(B) \ll_{p_{1}, \ldots, p_{k}} f_{1}(B)$.

- The Grassmannian $\mathbb{G}(k, n)$ of $k$-dimensional linear subspaces of $\mathbb{P}^{n}$ is assumed to be embedded into projective space by the Plücker embedding. In particular, we identify $\mathbb{G}(n-1, n)$ with the dual projective space $\mathbb{P}^{n *}$. The height of a rational linear subspace $\Lambda \subset \mathbb{P}^{n}$ is by definition the height of its Plücker coordinates. According to [6, Chapter I, Corollary 5I], we have

$$
H(\Lambda) \asymp_{n} \operatorname{det}(\boldsymbol{\Lambda})
$$

where

$$
\boldsymbol{\Lambda}=\left\{\mathbf{x} \in \mathbb{Z}^{n+1}:[\mathbf{x}] \in \Lambda\right\} \cup\{\mathbf{0}\}
$$

is the lattice associated to $\Lambda$ and $\operatorname{det}(\boldsymbol{\Lambda})$ is the volume of a fundamental domain of $\boldsymbol{\Lambda}$. 


\subsection{Results from the Geometry of Numbers}

The following result is well-known [4, Lemma 1(iii)]. It is one of the principal results from the subject known as the Geometry of Numbers, and it is one of the key tools in the proof of Theorem 1.

Lemma 2.1. Let $\boldsymbol{\Lambda} \subset \mathbb{Z}^{n}$ be a lattice of dimension $m$. Then $\boldsymbol{\Lambda}$ has a basis $\mathbf{b}_{1}, \ldots, \mathbf{b}_{m}$ such that if one writes $\mathbf{x} \in \mathbf{\Lambda}$ as $\mathbf{x}=\sum_{j} \lambda_{j} \mathbf{b}_{j}$, then

$$
\lambda_{j} \ll_{n}|\mathbf{x}| /\left|\mathbf{b}_{j}\right|
$$

Moreover one has

$$
\operatorname{det}(\boldsymbol{\Lambda}) \asymp_{n} \prod_{j=1}^{m}\left|\mathbf{b}_{j}\right| .
$$

The following result is a consequence of [6, Chapter I, Corollary 5J].

Lemma 2.2. Suppose that $\mathbf{a}_{1}, \ldots, \mathbf{a}_{k}$ are linearly independent $n$-dimensional vectors with integer components and let

$$
\mathbf{\Lambda}=\left\{\mathbf{x} \in \mathbb{Z}^{n}: \mathbf{a}_{1} \cdot \mathbf{x}=\cdots=\mathbf{a}_{k} \cdot \mathbf{x}=0\right\} .
$$

Then $\boldsymbol{\Lambda} \subset \mathbb{Z}^{n}$ is a lattice of dimension $n-k$ and

$$
\operatorname{det}(\boldsymbol{\Lambda}) \ll_{n} \prod_{j=1}^{k}\left|\mathbf{a}_{j}\right|
$$

\section{$2.2 \quad$ Bad linear sections}

The homogeneous ideal of a hypersurface $X \subset \mathbb{P}^{n}$ of degree $d$ is generated by a single homogeneous polynomial $F\left(Z_{0}, \ldots, Z_{n}\right)$ of degree $d$. This means that hypersurfaces in $\mathbb{P}^{n}$ of degree $d$ are parametrised by points in $\mathbb{P}\left(\overline{\mathbb{Q}}\left[Z_{0}, \ldots, Z_{n}\right]_{d}\right)$, where $\overline{\mathbb{Q}}\left[Z_{0}, \ldots, Z_{n}\right]_{d}$ is the vector space of homogeneous polynomials of degree $d$ in $n+1$ variables. Let $V(F)$ denote the hypersurface in $\mathbb{P}^{n}$ given by the zero locus of $F \in \overline{\mathbb{Q}}\left[Z_{0}, \ldots, Z_{n}\right]_{d}$. The set of pairs $(\Lambda, F) \in \mathbb{G}(k, n) \times \mathbb{P}\left(\overline{\mathbb{Q}}\left[Z_{0}, \ldots, Z_{n}\right]_{d}\right)$ for which $\Lambda \cap V(F)$ is an irreducible variety of dimension $k-1$ and degree $d$ is an open subset of $\mathbb{G}(k, n) \times \mathbb{P}\left(\overline{\mathbb{Q}}\left[Z_{0}, \ldots, Z_{n}\right]_{d}\right)$. We denote the complement of this open subset by $\Phi_{n, d, k}$.

The following result is well-known but the proof is so short that we reproduce it here.

Lemma 2.3. Let $X \subset \mathbb{P}^{n}$ be an irreducible hypersurface of degree $d$ and dimension at least two. Then the set of hyperplanes $\Gamma$ for which the linear section $\Gamma \cap X$ is reducible or of degree less than $d$ is a proper closed subset of $\mathbb{P}^{n *}$. Furthermore, this closed subset is cut out by hypersurfaces of degrees bounded solely in terms of $n$ and $d$. The number of required hypersurfaces is also bounded in terms of $n$ and $d$. 
Proof. By choosing a basis of $\overline{\mathbb{Q}}\left[Z_{0}, \ldots, Z_{n}\right]_{d}$, we may identify $\mathbb{P}\left(\overline{\mathbb{Q}}\left[Z_{0}, \ldots, Z_{n}\right]_{d}\right)$ with $\mathbb{P}^{N}$ for some $N$. Suppose that the ideal of $\Phi_{n, d, n-1} \subset \mathbb{P}^{n *} \times \mathbb{P}^{N}$ is generated by bihomogeneous polynomials

$$
G_{i}\left(Z_{0}, \ldots, Z_{n} ; W_{0}, \ldots, W_{N}\right) \quad \text { for } \quad i=1,2, \ldots, m .
$$

The set of hyperplanes $\Gamma$ for which $\Gamma \cap X$ is reducible or of degree less than $d$ is then the common zero locus of the polynomials $(2.1)$, where $W_{0}, \ldots, W_{N}$ are the coefficients of any homogeneous polynomial generating the ideal of $X$. Since a general hyperplane section $\Gamma \cap X$ is irreducible [3, Proposition 18.10], all of the polynomials (2.1) cannot vanish identically on $\mathbb{P}^{n *}$.

Lemma 2.4. Let $X \subset \mathbb{P}^{4}$ be an irreducible hypersurface of degree $d$ and let $V \subset \mathbb{P}^{4 *}$ be the set of hyperplanes $\Gamma$ with the following property. There is a point $p \in \Gamma \cap X$ such that for every two-plane $\Lambda \subset \Gamma$ either $\Lambda$ is contained in $X$ or $\Lambda \cap X$ contains an irreducible component of degree less than $d$. Then we have the following.

(a) $V$ is a closed subset of $\mathbb{P}^{4 *}$, and if $V \neq \mathbb{P}^{4 *}$, then $V$ is cut out by hypersurfaces of degrees bounded in terms of $d$. The number of required hypersurfaces is also bounded in terms of $d$.

(b) If $Y=\Gamma \cap X$ is an irreducible surface for some hyperplane $\Gamma \in V$, then $Y$ is a cone over a plane curve.

(c) $V=\mathbb{P}^{4 *}$ if and only if $X$ is a cone over a plane curve with respect to a vertex line.

Proof. As in the proof of Lemma 2.3 , we identify $\mathbb{P}\left(\overline{\mathbb{Q}}\left[Z_{0}, \ldots, Z_{4}\right]_{d}\right)$ with $\mathbb{P}^{N}$. Let $\Psi \subset \mathbb{P}^{4 *} \times \mathbb{P}^{4} \times \mathbb{P}^{N} \times \mathbb{G}(2,4)$ be the set of four-tuples $(\Gamma, p, F, \Lambda)$ such that $F(p)=0, p \in \Lambda, \Lambda \subset \Gamma$, and $(\Lambda, F) \in \Phi_{4, d, 2}$. Let $\pi$ be the projection map from $\Psi$ to $\mathbb{P}^{4 *} \times \mathbb{P}^{4} \times \mathbb{P}^{N}$. Then $\Psi$ is a projective variety and the function

$$
\lambda(q)=\operatorname{dim}\left(\pi^{-1}(q)\right)
$$

is an upper-semicontinuous function on the image $\pi(\Psi)$ [3, Corollary 11.13]. In particular,

$$
\Omega=\{(\Gamma, p, F) \in \pi(\Psi): \lambda(\Gamma, p, F) \geq 2\}
$$

is a subvariety of $\mathbb{P}^{4 *} \times \mathbb{P}^{4} \times \mathbb{P}^{N}$. Now the set of two-planes $\Lambda \subset \mathbb{P}^{4}$ for which $p \in \Lambda$ and $\Lambda \subset \Gamma$ for some $(\Gamma, p) \in \mathbb{P}^{4 *} \times \mathbb{P}^{4}$ is a two-dimensional linear subspace of $\mathbb{G}(2,4)$. The fibre $\pi^{-1}(\Gamma, p, F)$ is contained in this linear subspace. Hence, $\Omega$ is the set of triples $(\Gamma, p, F)$ such that $F(p)=0$ and such that $\Lambda \subset V(F)$ or $\Lambda \cap V(F)$ contains an irreducible component of degree less than $d$ for every two-plane $\Lambda \subset \Gamma$ containing $p$. Let $\Sigma$ be the projection of $\Omega$ on $\mathbb{P}^{4 *} \times \mathbb{P}^{N}$, and let

$$
G_{i}\left(Z_{0}, \ldots, Z_{n} ; W_{0}, \ldots, W_{N}\right) \quad \text { for } \quad i=1,2, \ldots, m,
$$

be bihomogeneous polynomials generating the ideal of $\Sigma$. If $W_{0}, \ldots, W_{N}$ are the coefficients of some homogeneous polynomial generating the ideal of $X$, then $V$ is the common zero locus of the polynomials (2.2). This proves (a). 
Next we consider (b). Let $Y=\Gamma \cap X$ be an irreducible hyperplane section and assume that $p$ is a point of $Y$ such that $\Lambda \cap X$ contains an irreducible component of degree less than $d$ for every two-plane $\Lambda \subset \Gamma$ containing $p$. Let $\pi: \widetilde{Y} \rightarrow Y$ be the blow-up of $Y$ at $p$. There is, then, a unique map $\psi: \widetilde{Y} \rightarrow \mathbb{P}^{2}$ which extends the projection map $Y \rightarrow \mathbb{P}^{2}$ from $p$ to some two-plane $\mathbb{P}^{2} \subset \Gamma$. If $\psi$ is surjective, then $\psi^{-1}(L)$ is irreducible of degree $d$ for a general line $L \subset \mathbb{P}^{2}$ [2, Theorem 1.1]. This contradicts the assumption that $\pi\left(\psi^{-1}(L)\right)$ is reducible for every line $L \subset \mathbb{P}^{2}$. Hence, the map $\psi$ is not surjective, so $Y$ is cone over a plane curve with vertex $p$.

To prove (c) we assume that $V=\mathbb{P}^{4 *}$ and consider the incidence correspondence $\Omega \subset \mathbb{P}^{4 *} \times X$ consisting of all pairs $(\Gamma, p)$ such that $\Lambda \subset X$ or $\Lambda \cap X$ contains an irreducible component of degree less than $d$ for every two-plane $\Lambda \subset \Gamma$ containing $p$. It follows from the proof of (a) that $\Omega$ is a closed subset of $\mathbb{P}^{4 *} \times X$. Since $V$ is the projection of $\Omega \subset \mathbb{P}^{4 *} \times X$ on the first factor, we have that the dimension of $\Omega$ is at least four. According to (b), we can then find a point $p \in X$ and a family of hyperplanes $\left\{\Gamma_{\lambda}\right\}$ through $p$ such that $\Gamma_{\lambda} \cap X$ are all cones with the common vertex $p$. It follows that $X$ is a cone with vertex $p$ over $\Gamma \cap X$ for any hyperplane $\Gamma \subset \mathbb{P}^{4}$ which does not contain $p$. Let $\Gamma \subset \mathbb{P}^{4}$ be such that $\Gamma \cap X$ is a cone over a plane curve $C \subset X$ with vertex $q \in X$. Then $X$ is a cone over $C$ with two different vertices $p$ and $q$. This proves the first implication of (c). The other one is immediate.

\subsection{Linear subspaces of hypersurfaces}

In this section we state some elementary results about linear subspaces contained in a hypersurface $X \subset \mathbb{P}^{n}$. Let $F_{k}(X) \subset \mathbb{G}(k, n)$ denote the Fano variety of $k$ planes contained in the variety $X \subset \mathbb{P}^{n}$. It can be shown that the number of irreducible components and the dimensions of the irreducible components of $F_{k}(X)$ can be bounded in terms of $d$.

Lemma 2.5. Let $X \subset \mathbb{P}^{n}$ be an irreducible hypersurface and assume that $X$ is not a hyperplane. Then the dimension of $F_{n-2}(X)$ is at most one and $F_{n-2}(X)$ contains no lines.

Proof. Suppose that $Y$ is an irreducible component of $F_{n-2}(X)$ of dimension at least one. Then the variety $\bigcup_{\Lambda \in Y} \Lambda$ has dimension at least $n-1$ and is therefore equal to $X$. In particular, every point on $X$ belongs to an $(n-2)$-plane $\Lambda$ in $Y$. Consider the incidence correspondence

$$
\Psi=\{(p, \Lambda) \in X \times Y: p \in \Lambda\} .
$$

The fibre of $\Psi$ over an $(n-2)$-plane $\Lambda$ is irreducible of dimension $n-2$. The variety $\Psi$ is therefore irreducible of dimension $\operatorname{dim}(Y)+n-2$ [3, Theorem 11.14]. Now if an $(n-2)$-plane $\Lambda \subset X$ contains the point $p$, then $\Lambda$ must lie in the projective tangent space $\mathbb{T}_{p}(X)$ of $X$ at $p$. For a non-singular point $p \in X$, the dimension of $X \cap \mathbb{T}_{p}(X)$ is $n-2$, so the fibre of $\Psi$ over a general point of $X$ is finite. The dimension of $\Psi$ is thus at most $n-1$. Hence, the dimension of $Y$ is 
at most one. To finish the proof we note that $\bigcup_{\Lambda \in Y} \Lambda$ is a hyperplane when $Y$ is a line. Since $X$ is irreducible, $F_{n-2}(X)$ cannot contain any lines.

The following lemma is a modification of Example 19.11 on page 244 in [3].

Lemma 2.6. Let $X \subset \mathbb{P}^{n}$ be the surface swept out by the lines parametrised by an irreducible curve $C \subset \mathbb{G}(1, n)$. Then the degree of $X$ does not exceed the degree of $C$.

Proof. The degree of $X$ is by definition the cardinality of the intersection $\Lambda \cap X$ for a general $(n-2)$-plane $\Lambda \subset \mathbb{P}^{n}$. Assume that $\Lambda \cap X$ contains $\operatorname{deg}(X)$ points. Now every point of $\Lambda \cap X$ belongs to a line $L \in C$ that meets $\Lambda$. The locus of lines $L \in \mathbb{G}(1, n)$ that meet $\Lambda$ is a hyperplane section $\Gamma \cap \mathbb{G}(1, n)$. If $C$ is contained in $\Gamma$, then every line $L \in C$ meet $\Lambda$. That is, we have a regular map $C \rightarrow \Lambda$ given by $L \mapsto L \cap \Lambda$. But $C$ is irreducible so the image of this map is irreducible. Hence, $\Lambda \cap X$ contains only one point so that the degree of $X$ is one. If $C$ is not contained in $\Gamma$, then there are at most $\operatorname{deg}(C)$ points in $\Gamma \cap C$. Hence, $\Lambda \cap X$ contains at most $\operatorname{deg}(C)$ points.

Lemma 2.7. Let $X \subset \mathbb{P}^{4}$ be an irreducible hypersurface of degree $d$, and let $C(p, X)$ be union of lines on $X$ passing through $p \in X$. Then the number of irreducible components and the degrees of the irreducible components of $C(p, X)$ can be bounded in terms of $d$.

Proof. Let $P_{i j}$, for $0 \leq i<j \leq 4$, be the Plücker coordinates on $\mathbb{G}(1,4)$, and assume that $p=[1,0,0,0,0]$. The lines in $\mathbb{P}^{4}$ that pass through $p$ are then parametrised by the points in the three-dimensional plane $\Lambda \subset \mathbb{G}(1,4)$ which is defined by

$$
P_{i j}=0 \text { for } \quad 0<i<j \leq 4 .
$$

The map $\phi: C(p, X) \backslash p \rightarrow \Lambda$, sending a point $q$ to the line through $p$ and $q$, is given by

$$
\left[Z_{0}, Z_{1}, Z_{2}, Z_{3}, Z_{4}\right] \mapsto\left[Z_{1}, Z_{2}, Z_{3}, Z_{4}, 0,0, \ldots\right]
$$

The map $\phi$ can thus be identified with the projection $\pi$ of $C(p, X) \backslash p$ to the hyperplane $Z_{0}=0 \mathrm{in} \mathbb{P}^{n}$. Under this identification, the image of $\pi$ is equal to $\Lambda \cap F_{1}(X)$. Since the number of irreducible components and the degrees of the irreducible components of $F_{1}(X)$ are bounded in terms of $d$, the same is true for $\Lambda \cap F_{1}(X)$ and $C(p, X)$.

\subsection{Estimates for counting functions}

It this section we list those known estimates for counting functions that we use in the proof of Theorem 1 .

(E1) Let $\Lambda$ be a $k$-dimensional linear subspace of $\mathbb{P}^{n}$. If $\Lambda$ contains $k+1$ linearly independent rational points of height at most $B$, then $\Lambda$ is defined over $\mathbb{Q}$ and

$$
N(\Lambda, B) \ll_{n} \frac{B^{k+1}}{H(\Lambda)} .
$$


To see this, let $\mathbf{b}_{1}, \ldots, \mathbf{b}_{k}$ be a basis of the lattice

$$
\boldsymbol{\Lambda}=\left\{\mathbf{x} \in \mathbb{Z}^{n+1}:[\mathbf{x}] \in \Lambda\right\} \cup\{\mathbf{0}\}
$$

with the properties stated in Lemma 2.1. Since $\Lambda$ contains $k+1$ linearly independent rational points of height at most $B$, we must have $\left|\mathbf{b}_{i}\right| \ll_{n} B$. Hence,

$$
N(\Lambda, B) \ll_{n} \frac{B^{k+1}}{\left|\mathbf{b}_{0}\right| \cdots\left|\mathbf{b}_{k}\right|} \asymp_{n} \frac{B^{k+1}}{H(\Lambda)}
$$

(E2) If $X \subset \mathbb{P}^{n}$ is an irreducible variety of degree $d$ and dimension $r$, then

$$
N(X, B) \ll_{n, d} B^{r+1} .
$$

This is proved for hypersurfaces in [4, Theorem 1]. The general result follows by a standard projection argument (see for example the proof of Lemma 1 in [1]).

(E3) If $X \subset \mathbb{P}^{n}$ is an irreducible variety of degree $d \geq 2$ and dimension $r$, then

$$
N(X, B) \ll_{n, d, \varepsilon} B^{r+1 / d+\varepsilon},
$$

for every $\varepsilon>0[5]$.

(E4) If $X \subset \mathbb{P}^{n}$ is an irreducible curve of degree $d$, then

$$
N(X, B) \ll_{n, d, \varepsilon} B^{2 / d+\varepsilon},
$$

for every $\varepsilon>0$. This estimate is proved for plane curves in [4, Theorem 3]. As in (E2), the general estimate follows by a projection argument.

(E5) Let $\Lambda \subset \mathbb{P}^{n}$ be a two-plane which is defined over the rational numbers. If $X \subset \Lambda$ is a non-singular curve of degree $d \geq 2$, then

$$
N(X, B) \ll_{n, d, \varepsilon} 1+\frac{B^{2 / d+\varepsilon}}{H(\Lambda)^{2 / 3 d}},
$$

for every $\varepsilon>0$. This follows from Theorem 3 and Lemma 1(iii) of [4].

(E6) If $X \subset \mathbb{P}^{n}$ is an irreducible surface of degree $d \geq 2$, then

$$
N(X, B) \ll_{n, d, \varepsilon} B^{2+\varepsilon},
$$

for every $\varepsilon>0[1$, Lemma 1$]$.

(E7) If $X \subset \mathbb{P}^{n}$ is a quadratic hypersurface of rank at least three, then

$$
N(X, B) \ll_{n, \varepsilon} B^{n-1+\varepsilon},
$$

for every $\varepsilon>0[4$, Theorem 2] 


\section{Proof of Theorem 1}

The idea of the proof is simple. We cover the set

$$
\left\{p \in \mathbb{P}^{4}(\mathbb{Q}): H(p) \leq B\right\}
$$

by a finite collection $I$ of linear subspaces $\Lambda \subset \mathbb{P}^{4}$, and put

$$
\Sigma=\bigcup_{\Lambda \in I}(\Lambda \cap X) .
$$

We then have

$$
N(X, B)=N(\Sigma, B) \quad \text { and } \quad \operatorname{dim}(X)>\operatorname{dim}(\Sigma) .
$$

We may thus apply the sharp estimates (E4) and (E6) from Section 2.4. To determine a suitable set $I$, we apply the results from Section 2.1.

Let $p=\left[Z_{0}, \ldots, Z_{4}\right]$ be a point of $\mathbb{P}^{4}$ such that $Z_{0}, \ldots, Z_{4}$ are relatively prime integers. According to [4, Lemma $1(\mathrm{i})]$, the set

$$
\boldsymbol{\Lambda}_{1}=\left\{\left(x_{0}, \ldots, x_{4}\right) \in \mathbb{Z}^{5}: Z_{0} x_{0}+\cdots+Z_{4} x_{4}=0\right\}
$$

is a lattice of dimension four and

$$
\operatorname{det}\left(\boldsymbol{\Lambda}_{1}\right)=\sqrt{Z_{0}^{2}+\cdots+Z_{4}^{2}} \asymp H(p) .
$$

Lemma 2.1 states that there exists a basis $\mathbf{b}_{1}, \mathbf{b}_{2}, \mathbf{b}_{3}, \mathbf{b}_{4}$ of $\boldsymbol{\Lambda}_{1}$ such that

$$
\left|\mathbf{b}_{1}\right|\left|\mathbf{b}_{2}\right|\left|\mathbf{b}_{3}\right|\left|\mathbf{b}_{4}\right| \asymp \operatorname{det}\left(\boldsymbol{\Lambda}_{1}\right) .
$$

Without loss of generality we may assume that

$$
\left|\mathbf{b}_{1}\right|\left|\mathbf{b}_{2}\right| \ll \operatorname{det}\left(\boldsymbol{\Lambda}_{1}\right)^{1 / 2} .
$$

Let

$$
\boldsymbol{\Lambda}_{2}=\left\{\mathbf{x} \in \mathbb{Z}^{5}: \mathbf{b}_{1} \cdot \mathbf{x}=\mathbf{b}_{2} \cdot \mathbf{x}=0\right\}
$$

and apply Lemma 2.1 again to find a basis $\mathbf{x}_{1}, \mathbf{x}_{2}, \mathbf{x}_{3}$ of the lattice $\boldsymbol{\Lambda}_{2}$ such that

$$
\left|\mathbf{x}_{1}\right|\left|\mathbf{x}_{2}\right|\left|\mathbf{x}_{3}\right| \asymp \operatorname{det}\left(\boldsymbol{\Lambda}_{2}\right) \ll\left|\mathbf{b}_{1}\right|\left|\mathbf{b}_{2}\right| .
$$

The last inequality of (3.1) follows from Lemma 2.2. Finally let

$$
\Lambda_{3}=\left\{\mathbf{a} \in \mathbb{Z}^{5}: \mathbf{a} \cdot \mathbf{x}_{1}=\mathbf{a} \cdot \mathbf{x}_{2}=\mathbf{a} \cdot \mathbf{x}_{3}=0\right\}
$$

and apply Lemma 2.1 to find a basis $\mathbf{a}_{1}, \mathbf{a}_{2}$ of $\boldsymbol{\Lambda}_{3}$ such that

$$
\left|\mathbf{a}_{1}\right|\left|\mathbf{a}_{2}\right| \asymp \operatorname{det}\left(\boldsymbol{\Lambda}_{3}\right) \ll\left|\mathbf{x}_{1}\right|\left|\mathbf{x}_{2}\right|\left|\mathbf{x}_{3}\right| .
$$

Then $\left(Z_{0}, \ldots, Z_{4}\right) \in \mathbf{\Lambda}_{2}$,

$$
\boldsymbol{\Lambda}_{2}=\left\{\mathbf{x} \in \mathbb{Z}^{5}: \mathbf{a}_{1} \cdot \mathbf{x}=\mathbf{a}_{2} \cdot \mathbf{x}=0\right\},
$$


and,

$$
\left|\mathbf{a}_{1}\right|\left|\mathbf{a}_{2}\right| \asymp \operatorname{det}\left(\boldsymbol{\Lambda}_{2}\right) \ll H(p)^{1 / 2} .
$$

This shows that there exists a rational two-plane $\Lambda \subset \mathbb{P}^{4}$ containing $p$ such that $H(\Lambda) \ll H(p)^{1 / 2}$. It also shows that $\Lambda=\Gamma_{1} \cap \Gamma_{2}$ for some rational hyperplanes $\Gamma_{1}, \Gamma_{2}$ in $\mathbb{P}^{4}$ such that $H\left(\Gamma_{1}\right) H\left(\Gamma_{2}\right) \asymp H(\Lambda)$.

Let $A$ be a positive constant and let $I \subset \mathbb{P}^{4 *}(\mathbb{Q}) \times \mathbb{P}^{4 *}(\mathbb{Q})$ be the set of pairs $\left(\Gamma_{1}, \Gamma_{2}\right)$ of hyperplanes such that

(i) $\Gamma_{1} \neq \Gamma_{2}$,

(ii) $H\left(\Gamma_{1}\right) H\left(\Gamma_{2}\right) \leq A H\left(\Gamma_{1} \cap \Gamma_{2}\right)$, where $\Gamma_{1} \cap \Gamma_{2}$ is considered as an element of $\mathbb{G}(2,4)$,

(iii) $H\left(\Gamma_{1}\right) \leq A B^{1 / 4}$, and

(iv) $H\left(\Gamma_{2}\right) \leq A B^{1 / 2} / H\left(\Gamma_{1}\right)$.

Provided that $A$ is large enough, we have

$$
\left\{p \in \mathbb{P}^{4}(\mathbb{Q}): H(p) \leq B\right\} \subset \bigcup_{\left(\Gamma_{1}, \Gamma_{2}\right) \in I}\left(\Gamma_{1} \cap \Gamma_{2}\right) .
$$

From the discussion above it follows that we may choose $A$ independently of $B$. This defines $I$ and $\Sigma$.

The next step of the proof is to use the estimates from Section 2.4 to estimate $N(\Sigma, B)$. The set $I$ can be partitioned into three subsets:

$I_{1}$ is the set of $\left(\Gamma_{1}, \Gamma_{2}\right) \in I$ such that $\Gamma_{1} \cap X$ contains an irreducible component of degree less than $d$.

$I_{2}$ is the set of $\left(\Gamma_{1}, \Gamma_{2}\right) \in I$ such that $\Gamma_{1} \cap X$ is irreducible of degree $d$ but $\Gamma_{1} \cap \Gamma_{2} \cap X$ contains an irreducible component of degree less than $d$.

$I_{3}$ is the set of $\left(\Gamma_{1}, \Gamma_{2}\right) \in I$ such that $\Gamma_{1} \cap \Gamma_{2} \cap X$ is an irreducible curve of degree $d$.

Let

$$
\Sigma_{i}=\bigcup_{\left(\Gamma_{1}, \Gamma_{2}\right) \in I_{i}}\left(\Gamma_{1} \cap \Gamma_{2} \cap X\right) \quad \text { for } \quad i=1,2,3 .
$$

Then

$$
N(\Sigma, B) \leq N\left(\Sigma_{1}, B\right)+N\left(\Sigma_{2}, B\right)+N\left(\Sigma_{3}, B\right) .
$$

\section{$3.1 \quad$ Estimate of $N\left(\Sigma_{1}, B\right)$}

Let $J$ be the set of rational hyperplanes $\Gamma \subset \mathbb{P}^{4}$ such that $H(\Gamma) \leq A B^{1 / 4}$ and such that $\Gamma \cap X$ is reducible or of degree less than $d=\operatorname{deg}(X)$. Consider an irreducible component $Y \subset \Gamma \cap X$ for some $\Gamma \in J$. If $Y$ is not a two-plane, then

$$
N(Y, B) \ll_{d, \varepsilon} B^{2+\varepsilon}
$$


according to (E6). If $Y$ is a two-plane such that all points of height at most $B$ on $\mathbb{P}^{4}(\mathbb{Q}) \cap Y$ lie on a line, then

$$
N(Y, B) \ll B^{2}
$$

according to (E2). Hence,

$$
N\left(\Sigma_{1}, B\right) \ll_{d, \varepsilon} B^{2+\varepsilon}|J|+N^{\prime}(X, B),
$$

where $N^{\prime}(X, B)$ is the number of rational points on $\mathbb{P}^{4}$ of height at most $B$ lying on the union of all two-planes in $X$ that contain three non-collinear rational points on $\mathbb{P}^{4}$ of height at most $B$. By Lemma 2.3 and (E2), the cardinality of $J$ is $O_{d}(B)$ so the first term of $(3.2)$ is $O_{d, \varepsilon}\left(B^{3+\varepsilon}\right)$. By the following lemma, the second term is also $O_{d, \varepsilon}\left(B^{3+\varepsilon}\right)$.

Lemma 3.1. Let $N^{\prime}(X, B)$ be the number of rational points on $\mathbb{P}^{4}$ of height at most $B$ lying on the union of all two-planes in $X$ that contain three non-collinear rational points on $\mathbb{P}^{4}$ of height at most $B$. Then,

$$
N^{\prime}(X, B) \ll_{d, \varepsilon} B^{3+\varepsilon} .
$$

Moreover, if $X$ is a cone with respect to two different rational vertex points of height at most $B$, then

$$
N(X, B) \ll_{d, \varepsilon} B^{3+\varepsilon} .
$$

Proof. If a two-plane $\Lambda \subset X$ contains three non-collinear rational points on $\mathbb{P}^{4}$ of height at most $B$, then $H(\Lambda) \leq A^{\prime} B^{3}$ for some constant $A^{\prime}$. Furthermore, $\Lambda$ contains $O\left(B^{3} / H(\Lambda)\right)$ rational points of height at most $B$ according to (E1). Hence,

$$
N^{\prime}(X, B) \leq \sum_{\substack{\Lambda \in F_{2}(X)(\mathbb{Q}) \\ H(\Lambda) \leq A^{\prime} B^{3}}} \frac{B^{3}}{H(\Lambda)}
$$

where $F_{2}(X) \subset \mathbb{G}(2,4)$ is the Fano variety of two-planes in $X$. By Lemma 2.5, the dimension of $F_{2}(X)$ is at most one and $F_{2}(X)$ contains no lines. The number of $\Lambda \in F_{2}(X)(\mathbb{Q})$ with $H(\Lambda) \ll T$ for some $T \geq 1$ is thus $O_{d, \varepsilon}\left(T^{1+\varepsilon}\right)$ according to $(\mathrm{E} 2)$. Hence,

$$
\sum_{\substack{\Lambda \in F_{2}(X)(\mathbb{Q}) \\ T<H(\Lambda) \leq 2 T}} \frac{B^{3}}{H(\Lambda)} \ll_{d, \varepsilon} \frac{B^{3}}{T} T^{1+\varepsilon} \ll B^{3+\varepsilon}
$$

when $T \ll B^{3}$. By summing over dyadic intervals, we get

$$
N^{\prime}(X, B) \ll_{d, \varepsilon} B^{3+\varepsilon} .
$$

To prove the second statement, let $p$ and $q$ be two different rational points on $\mathbb{P}^{4}$ of height at most $B$ which are vertex points of $X$. If every point of $\mathbb{P}^{4}(\mathbb{Q}) \cap X$ of height at most $B$ belongs to the line $L$ containing $p$ and $q$, then

$$
N(X, B) \ll B^{2}
$$


according to $(\mathrm{E} 2)$. If there is some point of $\mathbb{P}^{4}(\mathbb{Q}) \cap X$ of height at most $B$ that does not belong to the line $L$, then every point of $\mathbb{P}^{4}(\mathbb{Q}) \cap X$ belongs to a two-plane in $X$ that contains three non-collinear rational points on $\mathbb{P}^{4}$ of height at most $B$. In that case

$$
N(X, B)=N^{\prime}(X, B) \ll_{d, \varepsilon} B^{3+\varepsilon}
$$

by what we just proved.

\subsection{Estimate of $N\left(\Sigma_{2}, B\right)$}

Consider an irreducible component $Y \subset \Gamma_{1} \cap \Gamma_{2} \cap X$ for some $\left(\Gamma_{1}, \Gamma_{2}\right) \in I_{2}$. If the degree of $Y$ is at least three, then

$$
N(Y, B) \ll_{d, \varepsilon} B^{2 / 3+\varepsilon}
$$

according to (E4). If the degree of $Y$ is two, then

$$
N(Y, B) \ll_{\varepsilon} 1+\frac{B^{1+\varepsilon}}{H\left(\Gamma_{1} \cap \Gamma_{2}\right)^{1 / 3}}
$$

according to (E5). If $Y$ is a line such that $Y$ contains at most one rational point of height at most $B$, then $N(Y, B) \leq 1$. Hence,

$$
\begin{aligned}
N\left(\Sigma_{2}, B\right) & \ll_{d, \varepsilon} B^{2 / 3+\varepsilon}\left|I_{2}\right| \\
& +\sum_{\left(\Gamma_{1}, \Gamma_{2}\right) \in I_{2}} \frac{B^{1+\varepsilon}}{H\left(\Gamma_{1} \cap \Gamma_{2}\right)^{1 / 3}} \\
& +\left|I_{2}\right|+N(Z, B),
\end{aligned}
$$

where $Z \subset X$ is the union of all lines $L \subset \Sigma_{2}$ that contain two different rational points of height at most $B$. Note that if $X$ is a cone over a plane curve, then $\Gamma_{1} \cap \Gamma_{2} \cap X$ is a union of lines for every pair $\left(\Gamma_{1}, \Gamma_{2}\right) \in I_{2}$. Thus, the first two terms of (3.3) do not appear in this case.

Lemma 3.2. We have

$$
\#\left\{\left(\Gamma_{1}, \Gamma_{2}\right) \in I_{2}: H\left(\Gamma_{i}\right) \leq T_{i}\right\} \ll_{d, \varepsilon} T_{1}^{5} T_{2}^{10 / 3+\varepsilon}+T_{1}^{5-\eta} T_{2}^{4},
$$

where $\eta=1$ unless $X$ is a cone over a plane curve in which case $\eta=0$.

Proof. Let $f: \Gamma_{1} \rightarrow \mathbb{P}^{3}$ be an isomorphism for some hyperplane $\Gamma_{1} \subset \mathbb{P}^{4}$ such that $\Gamma_{1} \cap X$ is irreducible. Let

$$
g: \mathbb{P}^{4 *} \backslash\left\{\Gamma_{1}\right\} \rightarrow \mathbb{P}^{3 *}
$$

be the projection map induced by $f^{-1}$. By Lemma 2.3, the set of two-planes $\Lambda \subset \mathbb{P}^{3}$ for which $\Lambda \cap f^{-1}(X)$ contains an irreducible component of degree less than $d=\operatorname{deg}(X)$ is a proper closed subset $V \subset \mathbb{P}^{3 *}$. The set of hyperplanes 
$\Gamma_{2} \in \mathbb{P}^{4 *} \backslash\left\{\Gamma_{1}\right\}$ for which $\Gamma_{1} \cap \Gamma_{2} \cap X$ is reducible is thus contained in the proper closed subset $W=\overline{g^{-1}(V)}$ of $\mathbb{P}^{4 *}$. There are two cases to consider.

If $V$ does not contain any two-planes, then $W$ does not contain any hyperplanes. Hence, the number of $\left(\Gamma_{1}, \Gamma_{2}\right) \in I_{2}$ with $\Gamma_{1}$ fixed and $H\left(\Gamma_{2}\right) \leq T_{2}$ is $O_{d, \varepsilon}\left(T_{2}^{10 / 3+\varepsilon}\right)$ by (E3) and (E7). Note that $W$ is cut out by $O_{d}(1)$ hypersurfaces of degrees $O_{d}(1)$ since $V$ is. The number of $\Gamma_{1} \in \mathbb{P}^{4 *}(\mathbb{Q})$ of height at most $T_{1}$ is $O\left(T_{1}^{5}\right)$. Hence, the number of pairs $\left(\Gamma_{1}, \Gamma_{2}\right) \in I_{2}$ such that $V$ does not contain any two-planes and $H\left(\Gamma_{i}\right) \leq T_{i}$ is $O_{d, \varepsilon}\left(T_{1}^{5} T_{2}^{10 / 3+\varepsilon}\right)$.

If $V$ contains a two-plane, then $W$ contains a hyperplane. The best estimate for the number of $\left(\Gamma_{1}, \Gamma_{2}\right) \in I_{2}$ with $\Gamma_{1}$ fixed and $H\left(\Gamma_{2}\right) \leq T_{2}$ is therefore $O_{d}\left(T_{2}^{4}\right)$. This is the trivial estimate (E2). Lemma 2.4 states that the set of $\Gamma_{1}$ for which $V$ contains a two-plane is contained in a hypersurface in $\mathbb{P}^{4 *}$ of degree $O_{d}(1)$, provided that $X$ is not a cone over a plane curve. In this case there are $O_{d}\left(T_{1}^{4}\right)$ such $\Gamma_{1} \in \mathbb{P}^{4 *}(\mathbb{Q})$ of height at most $T_{1}$, again according to (E2). In the general case we have $O\left(T_{1}^{5}\right)$ hyperplanes. Hence, the number of pairs $\left(\Gamma_{1}, \Gamma_{2}\right) \in I_{2}$ such that $V$ contains a two-plane and $H\left(\Gamma_{i}\right) \leq T_{i}$ is $O_{d}\left(T_{1}^{5-\eta} T_{2}^{4}\right)$, where $\eta=1$ unless $X$ is a cone over a plane curve in which case $\eta=0$.

We can use Lemma 3.2 to estimate the cardinality of $I_{2}$. The number of $\left(\Gamma_{1}, \Gamma_{2}\right) \in I_{2}$ with $T<H\left(\Gamma_{1}\right) \leq 2 T$ for some $T \ll B^{1 / 4}$ is

$$
\ll_{d, \varepsilon}\left(\frac{B^{1 / 2}}{T}\right)^{10 / 3+\varepsilon} T^{5}+\left(\frac{B^{1 / 2}}{T}\right)^{5-\eta} T^{4} \ll \begin{cases}B^{25 / 12+\varepsilon} & \text { if } \eta=1, \\ B^{5 / 2+\varepsilon} & \text { if } \eta=0 .\end{cases}
$$

By summing over dyadic intervals we get

$$
\left|I_{2}\right| \ll_{d, \varepsilon} \begin{cases}B^{25 / 12+\varepsilon} & \text { if } \eta=1, \\ B^{5 / 2+\varepsilon} & \text { if } \eta=0 .\end{cases}
$$

Consequently, the first term of (3.3) is $O_{d, \varepsilon}\left(B^{3+\varepsilon}\right)$.

In order to estimate the second term of (3.3) we divide the ranges of both $\Gamma_{1}$ and $\Gamma_{2}$ into dyadic intervals. If $T_{1} \ll B^{1 / 4}$ and $T_{1} T_{2} \ll B^{1 / 2}$, then

$$
\begin{aligned}
\sum_{\substack{\left(\Gamma_{1}, \Gamma_{2}\right) \in I_{2} \\
T_{i}<H\left(\Gamma_{i}\right) \leq 2 T_{i}}} \frac{B^{1+\varepsilon}}{H\left(\Gamma_{1} \cap \Gamma_{2}\right)^{1 / 3}} & \ll d, \varepsilon \frac{B^{1+\varepsilon}}{\left(T_{1} T_{2}\right)^{1 / 3}}\left(T_{1}^{5} T_{2}^{10 / 3+\varepsilon}+T_{1}^{4} T_{2}^{4}\right) \\
& \ll B^{35 / 12+\varepsilon} .
\end{aligned}
$$

Hence, the second term of (3.3) is $O_{d, \varepsilon}\left(B^{3+\varepsilon}\right)$.

We have already seen that $\left|I_{2}\right|=O_{d, \varepsilon}\left(B^{3+\varepsilon}\right)$, so it remains to estimate the very last term in (3.3). Let $Z_{1}(T)$ be the union of all lines $L \subset Z$ with $H(L)>T$, for some $T>0$, and let $Z_{2}(T)$ be the union of all lines $L \subset Z$ with $H(L) \leq T$. Then,

$$
N(Z, B) \leq N\left(Z_{1}(T), B\right)+N\left(Z_{2}(T), B\right),
$$

for every $T>0$. 
Lemma 3.3. If $T \leq B^{2}$, then

$$
N\left(Z_{1}(T), B\right) \ll_{d, \varepsilon} B^{13 / 4} T^{2 / d-1+\varepsilon} .
$$

Proof. Let $J \subset \mathbb{P}^{4 *}(\mathbb{Q})$ be the projection of $I_{2} \subset \mathbb{P}^{4 *}(\mathbb{Q}) \times \mathbb{P}^{4 *}(\mathbb{Q})$ on the first factor. If $\Gamma_{1} \in J$, then $\Gamma_{1} \cap X$ is an irreducible surface of degree $d$. By Lemma 2.5, the dimension of $F_{1}\left(\Gamma_{1} \cap X\right)$ is at most one, and by Lemma 2.6, every one-dimensional irreducible component of $F_{1}\left(\Gamma_{1} \cap X\right)$ has degree at least $d$. There are thus $O_{d, \varepsilon}\left(R^{2 / d+\varepsilon}\right)$ rational lines $L \subset \Gamma_{1} \cap X$ of height at most $R$ according to (E4). If the line $L$ is contained in $Z$, then $L$ contains two different rational points on $\mathbb{P}^{4}$ of height at most $B$. Hence,

$$
\sum_{\substack{L \in F_{1}\left(\Gamma_{1} \cap X\right)(\mathbb{Q}) \\ L \subset Z \\ R<H(L) \leq 2 R}} N(L, B) \ll_{d, \varepsilon} \frac{B^{2}}{R} R^{2 / d+\varepsilon} \ll B^{2} R^{2 / d-1+\varepsilon}
$$

according to (E1). The cardinality of $J$ is $O\left(B^{5 / 4}\right)$, so

$$
\sum_{\substack{L \subset Z \\ R<H(L) \leq 2 R}} N(L, B) \ll_{d, \varepsilon} B^{13 / 4} R^{2 / d-1+\varepsilon} .
$$

By summing over dyadic intervals, we get

$$
N\left(Z_{1}(T), B\right) \ll_{d, \varepsilon} B^{13 / 4} T^{2 / d-1+\varepsilon} .
$$

Note that we only have to sum over $O(\log B)$ dyadic intervals since $H(L) \ll B^{2}$ for every line $L$ that contains two rational points of height at most $B$.

Lemma 3.4. If $T \leq B^{2}$, then

$$
N\left(Z_{2}(T), B\right) \ll_{d, \varepsilon} B^{3+\varepsilon}+B^{2+\varepsilon} T^{(3+1 / d) / 2+\varepsilon} .
$$

Proof. Every rational line in $\mathbb{P}^{4}$ contains a rational point $p$ such that

$$
H(p) \ll A^{\prime \prime} H(L)^{1 / 2}
$$

for some positive constant $A^{\prime \prime}$. This is a consequence of Lemma 2.1 and 2.2. Hence,

$$
Z_{2}(T) \subset \bigcup_{\substack{p \in X(\mathbb{Q}) \\ H(p) \leq A^{\prime \prime} T}}(Z \cap C(p, X))
$$

where $C(p, X)$ is the cone of lines in $X$ with vertex $p$.

Suppose that $C(p, X)=X$ for some rational point $p \in X$ and let $J \subset \mathbb{P}^{4 *}(\mathbb{Q})$ be the projection of $I_{2} \subset \mathbb{P}^{4 *}(\mathbb{Q}) \times \mathbb{P}^{4 *}(\mathbb{Q})$ on the first factor. There are $O(B)$ hyperplanes $\Gamma_{1} \in J$ passing through $p$, and there are $O_{d, \varepsilon}\left(R^{2 / d+\varepsilon}\right)$ rational lines of height at most $R$ on the irreducible surface $\Gamma_{1} \cap X$ for any $\Gamma_{1} \in J$ (see the proof of Lemma 3.3). We are only interested in lines that contain two different 
points of height at most $B$, and on such lines there are $O\left(B^{2} / R\right)$ rational points on $\mathbb{P}^{4}$ of height at most $B$ if $R \leq B^{2}$. Hence,

$$
\sum_{\substack{L \subset Z \cap C(p, X) \\ R<H(p) \leq 2 R}} N(L, B) \ll_{d, \varepsilon} B^{3} R^{2 / d-1+\varepsilon}
$$

By summing over dyadic intervals, we get

$$
N(Z \cap C(p, X), B) \ll_{d, \varepsilon} B^{3+\varepsilon} .
$$

If there are two different rational points $p$ and $q$ on $\mathbb{P}^{4}$ of height at most $T^{1 / 2} \leq B$ such that

$$
X=C(p, X)=C(q, X)
$$

then

$$
N\left(Z_{2}(T), B\right) \leq N(X, B) \ll_{d, \varepsilon} B^{3+\varepsilon}
$$

according to Lemma 3.1. Hence, the contribution to $N\left(Z_{2}(T), B\right)$ from the points $p$ with $C(p, X)=X$ is $O_{d, \varepsilon}\left(B^{3+\varepsilon}\right)$.

Now assume that $C(p, X) \neq X$ for some rational point $p \in X$ and consider an irreducible component $Y \subset C(p, X)$. If $Y$ is not a two-plane, then

$$
N(Y, B) \ll_{d, \varepsilon} B^{2+\varepsilon}
$$

according to (E2) or (E6). Note that the degree of $Y$ is bounded in terms of $d$ according to Lemma 2.7. If $Y$ is a two-plane such that all points of height at most $B$ on $\mathbb{P}^{4}(\mathbb{Q}) \cap Y$ lie on a line, then

$$
N(Y, B) \ll B^{2}
$$

according to (E2). Hence,

$$
\sum_{\substack{p \in X(\mathbb{Q}) \\ H(p) \leq A^{\prime \prime} T^{1 / 2} \\ C(p, X) \neq X}} N(C(p, X), B) \ll_{d, \varepsilon} B^{2+\varepsilon} N\left(X, T^{1 / 2}\right)+N^{\prime}(X, B),
$$

where $N^{\prime}(X, B)$ is the cardinality defined in Lemma 3.1. The first term in (3.6) is $O_{d, \varepsilon}\left(B^{2+\varepsilon} T^{(3+1 / d) / 2+\varepsilon}\right)$ according to (E3), and the second term is $O_{d, \varepsilon}\left(B^{3+\varepsilon}\right)$ according to Lemma 3.1. Hence,

$$
N\left(Z_{2}(T), B\right) \ll_{d, \varepsilon} B^{3+\varepsilon}+B^{2+\varepsilon} T^{(3+1 / d) / 2+\varepsilon},
$$

provided that $T \ll B^{2}$.

If we put $T=B^{\frac{5 d}{10 d-6}}$ in (3.4) and apply Lemma 3.3 and 3.4 , then we get

$$
N(Z, B) \ll_{d, \varepsilon} B^{3+\varepsilon}+B^{\frac{11}{4}+\frac{7}{10 d-6}+\varepsilon} .
$$




\subsection{Estimate of $N\left(\Sigma_{3}, B\right)$}

Consider those pairs $\left(\Gamma_{1}, \Gamma_{2}\right) \in I_{3}$ with $T<H\left(\Gamma_{1}\right) \leq 2 T$ for some $T \ll B^{1 / 4}$. For each such pair we use Lemma 2.1 to find a basis $\mathbf{b}_{0}, \mathbf{b}_{1}, \mathbf{b}_{2}$ of the lattice

$$
\left\{\mathbf{x} \in \mathbb{Z}^{5}:[\mathbf{x}] \in \Gamma_{1} \cap \Gamma_{2}\right\} \cup\{\mathbf{0}\} .
$$

With out loss of generality, we may assume that

$$
\left|\mathbf{b}_{0}\right| \leq\left|\mathbf{b}_{1}\right| \leq\left|\mathbf{b}_{2}\right| .
$$

Let $\phi: \Gamma_{1} \cap \Gamma_{2} \rightarrow \mathbb{P}^{2}$ be the map

$$
\left[\lambda_{0} \mathbf{b}_{0}+\lambda_{1} \mathbf{b}_{1}+\lambda_{2} \mathbf{b}_{2}\right] \mapsto\left[\lambda_{0}, \lambda_{1}, \lambda_{2}\right]
$$

Then $H(\phi(p)) \ll H(p) /\left|\mathbf{b}_{0}\right|$ for a rational point $p \in \Gamma_{1} \cap \Gamma_{2}$, so

$$
N\left(\Gamma_{1} \cap \Gamma_{2} \cap X, B\right) \ll_{d, \varepsilon}\left(\frac{B}{\left|\mathbf{b}_{0}\right|}\right)^{2 / d+\varepsilon}
$$

according to (E4). Now consider all bases $\mathbf{b}_{0}, \mathbf{b}_{1}, \mathbf{b}_{2}$ which satisfy $C_{i}<\left|\mathbf{b}_{i}\right| \leq$ $2 C_{i}$ for some positive numbers $C_{i}$ with $C_{0} \ll C_{1} \ll C_{2}$. The set of $\Gamma \in \mathbb{P}^{4 *}$ which contains the point $\left[\mathbf{b}_{0}\right]$ is a hyperplane $\Lambda$ in $\mathbb{P}^{4 *}$, and the number of $\Gamma \in \Lambda(\mathbb{Q})$ with $H(\Gamma) \leq R$ is $O\left(R^{4} /\left|\mathbf{b}_{0}\right|\right)$, provided that $R \gg\left|\mathbf{b}_{0}\right|$ [4, Lemma $\left.1(\mathrm{v})\right]$. Hence, the number of pairs $\left(\Gamma_{1}, \Gamma_{2}\right) \in I_{3}$ with $T<H\left(\Gamma_{1}\right) \leq 2 T, C_{i}<\left|\mathbf{b}_{i}\right| \leq 2 C_{i}$, and $\mathbf{b}_{0}$ fixed is

$$
\ll\left(\frac{\left(\left(C_{0} C_{1} C_{2}\right) / T\right)^{4}}{C_{0}}\right)\left(\frac{T^{4}}{C_{0}}\right)=C_{0}^{2} C_{1}^{4} C_{2}^{4} .
$$

The number of $\mathbf{b}_{0}$ with $\left|\mathbf{b}_{0}\right| \ll C_{0}$ is $O\left(C_{0}^{5}\right)$. Hence,

$$
\begin{aligned}
& \sum_{\substack{\left(\Gamma_{1}, \Gamma_{2}\right) \in I_{3} \\
T<H\left(\Gamma_{1}\right) \leq 2 T \\
C_{i}<\left|\mathbf{b}_{i}\right| \leq 2 C_{i}}} N\left(\Gamma_{1} \cap \Gamma_{2} \cap X, B\right) \ll_{d, \varepsilon} C_{0}^{7-2 / d} C_{1}^{4} C_{2}^{4} B^{2 / d+\varepsilon} \\
& \ll\left(C_{0} C_{1} C_{2}\right)^{5-2 / 3 d} B^{2 / d+\varepsilon} \\
& \ll B^{5 / 2+5 / 3 d+\varepsilon} \text {. }
\end{aligned}
$$

By summing over dyadic intervals we get

$$
N\left(\Sigma_{3}, B\right) \ll_{d, \varepsilon} B^{5 / 2+5 / 3 d+\varepsilon} .
$$

\subsection{Conclusion}

We have shown that if $X \subset \mathbb{P}^{4}$ is an irreducible hypersurface of degree $d \geq 3$, then

$$
N(X, B) \ll_{d, \varepsilon} B^{3+\varepsilon}+B^{\frac{11}{4}+\frac{7}{10 d-6}+\varepsilon}+B^{\frac{5}{2}+\frac{5}{3 d}+\varepsilon},
$$

for every $\varepsilon>0$. It is straightforward to see that this estimate implies Theorem 1 . 


\section{References}

[1] T. D. Browning, A note on the distribution of rational points on threefolds, Quart. J. Math., 54 (2003), 33-39.

[2] W. Fulton and R. Lazarsfeld, Connectivity and its applications in algebraic geometry, Algebraic Geometry (LNM 862), Springer-Verlag, 1981, 26-92.

[3] J. Harris, Algebraic Geometry, Springer-Verlag, 1992.

[4] D. R. Heath-Brown, The density of rational points on curves and surfaces, Ann. of Math., 155 (2002), 553-595.

[5] J. Pila, Density of integral and rational points on varieties, Astérisque, 228 (1995), 183-187.

[6] W. M. Schmidt, Diophantine Approximations and Diophantine Equations (LNM 1467), Springer-Verlag, 1991. 